\title{
Kinetic moments method for the canonical ensemble distribution
}

\author{
William G. Hoover ${ }^{\text {a }}$, Brad Lee Holian ${ }^{b}$ \\ "Department of Applied Science. University of California at Dawis, Department of Physics, Lawrence Livermore National Laboratory, \\ Livermore, CA 94551-7808, USA \\ "Theoretical Dibision, Los Alamos Scienific National Laboratory, LoS Alamos, NM 87545, USA
}

Received 16 October 1995; accepted for publication 14 December 1995

Communicated by C.R. Doering

\begin{abstract}
Simultaneous integral control of the kinetic energy $\langle K\rangle$ and its fluctuation $\left\langle K^{2}\right\rangle-\langle K\rangle^{2}$ gives an extended phase-space distribution consistent with Gibbs' canonical one. This generalization of the Nosé-Hoover approach to thermostatting is the simplest time-reversible scheme to exhibit ergodicity for the one-dimensional harmonic oscillator. It is also applicable to equilibrium and nonequilibrium many-body simulations.
\end{abstract}

\section{Canonical dynamics}

Nosé discovered a dynamics consistent with Gibbs' canonical ensemble in 1984 [1]. His approach uses time-reversible integral feedback to control the kinetic energy. For a harmonic oscillator with unit mass and force constant, Hamilton's motion equations are

$\dot{q}=p, \quad \dot{p}=-q$.

Nosé's approach [1] adds temperature to the motion equations through a time-reversible friction coefficient $\zeta$, with an associated relaxation time $\tau$. Without loss of generality we consider specifically unit temperature, $k T=1$, for which the Gibbsian average value of the kinetic energy is $\frac{1}{2}$,

$\langle K\rangle=\left\langle\frac{1}{2} p^{2}\right\rangle=\frac{1}{2}$

A time-reversible frictional force $-\zeta p$ is introduced in order to control the kinetic energy. Applying the continuity equation for the probability density, of the flow equations,

$\dot{q}=p, \quad \dot{p}=-q-\zeta p, \quad \dot{\zeta}=\left(p^{2}-1\right) / \tau^{2}$,

in the "extended" phase-space $\{q, p, \zeta\}$, shows that the motion equations are consistent with an extended form of Gibbs' canonical distribution,

$f \propto \exp \left[-\frac{1}{2}\left(q^{2}+p^{2}+\tau^{2} \zeta^{2}\right)\right]$.

It is noteworthy that the flow equations are time reversible (with both $p$ and $\zeta$ changing sign in the reversed motion).

Numerical exploration of Nosé's motion equations [2], as well as some more-complicated relatives, reveals a fairly common lack of ergodicity, with the harmonic-oscillator phase space partitioned into separate regular and chaotic regions (Fig. 1). During the decade following Nosé's work many additional suggestions [3-11] were made with the intention of achieving ergodicity by using additional control variables.

Thermostats are, by and large;" ad "hoc'modifications of the equations of motion; though it is true that 
some satisfy variational principles, such as Gauss' principle of least constraint and Hamilton's principle of least action [12]. Aside from such variational bases, the only lasting criteria for the relative merits of the various approaches are Occam's, elegance and simplicity. We summarize previous work in Section 2 , and describe what seems to us the simplest approach to canonical ergodicity in Section 3 . We illustrate this kinetic moments method for three example problems. The final section summarizes our conclusions.

\section{Previous work}

Nosé's original description of his dynamics [1] introduced two distracting notions, "virtual" variables and time scaling, as consequences of his original approach through Hamiltonian mechanics. Hoover [2] emphasized the desirability of abandoning Nosé's Hamiltonian basis in order to avoid these unnecessary concepts. Nevertheless, the confusion associated with the virtual variables and time scaling persists.

Jellinek and Berry $[3,4]$ considered the most general Hamiltonian approach to the canonical distribution. Multiplicative scalings of coordinates, momenta, and time are all included. Their work is primarily formal, rather than numerical, and their approach excludes all known useful schemes. They suggest that the equivalence of time and phase averages for non-Hamiltonian approaches is problematic, though the basis for that claim is unclear. The nonHamiltonian example which we present in Section 3 provides an example of the time-average phase-average equivalence, and should prove helpful in evaluating the merits of the formal approaches.

Bauer, Bulgac, and Kusnezov [5] consider the thermostatting problem in rather different, but still quite general, terms, without insisting on a Hamiltonian basis. They provide a host of interesting worked-out examples. Their work, like that of Refs. $[4,6]$ considers additive, rather than multiplicative, terms in the equations of motion, and certainly includes our own favorite procedure as a special case. We regard additional additive forces as more physical than additions to the coordinate equation, $\dot{q}=p$. We use this distinction to motivate the present work.
Hamilton [7] emphasized the requirement that any reasonable modified equations of motion must still satisfy the virial theorem. It seems to us that this requirement is satisfied automatically by any dynamics which is consistent with Gibbs' distribution. Winkler [8] used a special time scaling, resembling Nosé's, but lacking in the additivity of the force contributions.

Martyna, Klein, and Tuckerman [9], introduced a "chain" of frictional variables, retaining the additivity of the thermostatting forces. Holian pointed out that this approach, while perfectly valid at equilibrium, is no longer effective in controlling the desired temperature, $\left\langle p^{2}\right\rangle=T$, away from equilibrium. Evans and Holian [11] demonstrated that many different thermostatting approaches predict very similar results in three-dimensional shear-flow simulations far from equilibrium.

The many varied approaches just reviewed suggest several desirable properties for themostatting (or control) forces:

(1) Time reversibility;

(2) Additivity, affecting the equation of motion for momentum;

(3) Ergodicity, providing the complete canonical distribution;

(4) Applicability away from equilibrium;

(5) Hamiltonian basis.

There is considerable evidence that nonequilibrium systems cannot be described by a straightforward generalization of Hamiltonian (or Lagrangian) mechanics, so that properties (4) and (5) may well be mutually exclusive. Our own approach is non-Hamiltonian and exhibits only the first four properties.

For applications, a sixth property, efficiency, should properly be considered. That is, an efficient approach should minimize the required computer time for a fixed standard of accuracy. This question has been studied extensively for Hamiltonian mechanics [13] as well as for shear flows far from equilibrium [11], comparing the relative accuracies of simple second-order integrators, more-complex higher-order multistep integrators, and the standard Runge-Kutta approach, all for a fixed number of force evaluations. Our own investigations of the equations discussed here lead to very similar conclusions, so that we do not address this important question further here. 
at any

;t still

It this

ynam-

Win

nbling

force

ced a

iditiv-

$\geq \mathrm{d}$ out

juilib-

lesired

brium.

ly dif-

imilar

lations

4 $\mathrm{Sug}$ -

tatting

motion

hor

quilibghtforangian) well be - Hamrties.

ciency. fficient imputer uestion an mefrom uracies omplex tandard aber of of the concluuportant

\section{Kinetic moments method}

If control of the kinetic energy does not provide the complete canonical distribution, then at least some moments of the kinetic energy must necessarily be in error [14]. This suggests controlling additional moments. For the harmonic oscillator, at unit temperature,

$\langle K\rangle=\frac{1}{2},\left\langle K^{2}\right\rangle=\frac{3}{4},\left\langle K^{3}\right\rangle=\frac{15}{8}, \ldots$.

The additional moments can be controlled independently, as described in Ref. [4] and Section 5.8 of Ref. [6]. For the first two moments, the corresponding control-variable equations are

$\dot{\zeta}=p^{2}-1, \quad \dot{\xi}=p^{4}-3 p^{2}$.
The two friction coefficients $\{\zeta, \xi\}$ can have independent relaxation times associated with them, as well as arbitrary weight functions, varying in time and space, without destroying the consistency with the canonical distribution. To explore the applicability of the method, we consider in turn (i) a one-dimensional harmonic oscillator, (ii) a many-body equilibrium crystal, and (iii) a nonequilibrium many-body shear flow.

\subsection{One-dimensional oscillator}

Solutions for three versions of the oscillator equations are shown in Fig. 1. The three cases are: (i) the original "Nosé-Hoover" oscillator, with $\zeta$ control of $K$; (ii) an oscillator with $\xi$ control of $K^{2}$; (iii) an
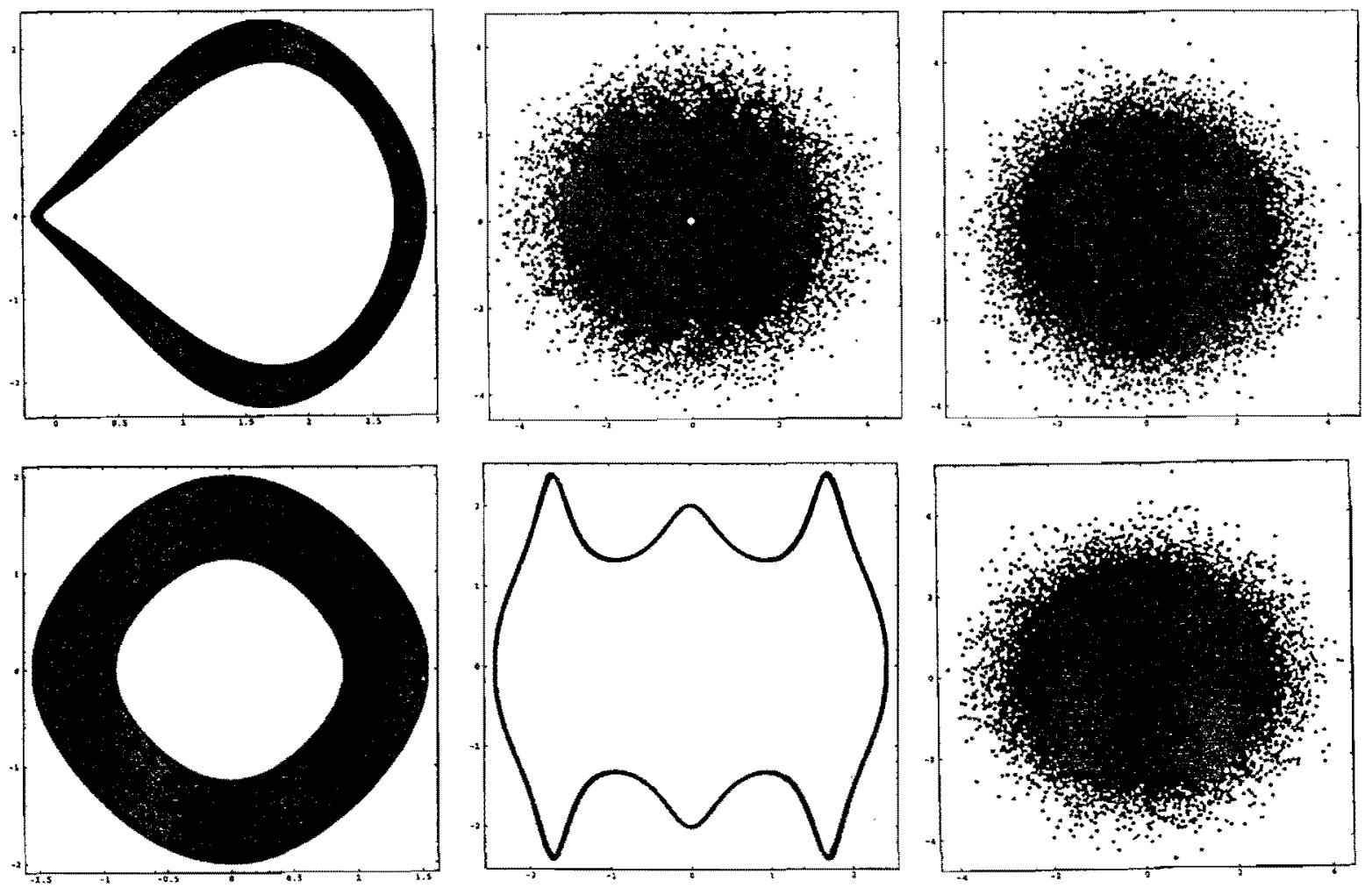

Fig. 1. $200000(q, p)$ trajectory points from simulations made up of 20000000 fourth-order Runge-Kutta time steps of length 0.005 . From left to right the trajectories were generated with $\langle K\rangle$ control, $\left\langle K^{2}\right\rangle$ control, and simultaneous $\langle K\rangle$ and $\left\langle K^{2}\right\rangle$ control. The initial condition for the upper row is $\{q, p, \zeta, \xi\}=\{2,2,0,0\}$, while that for the lower row is $\{0,2,0,0\}$. In all cases, the relaxation times, $\tau_{\xi}$ and $\tau_{\xi}$, were equal to unity. 


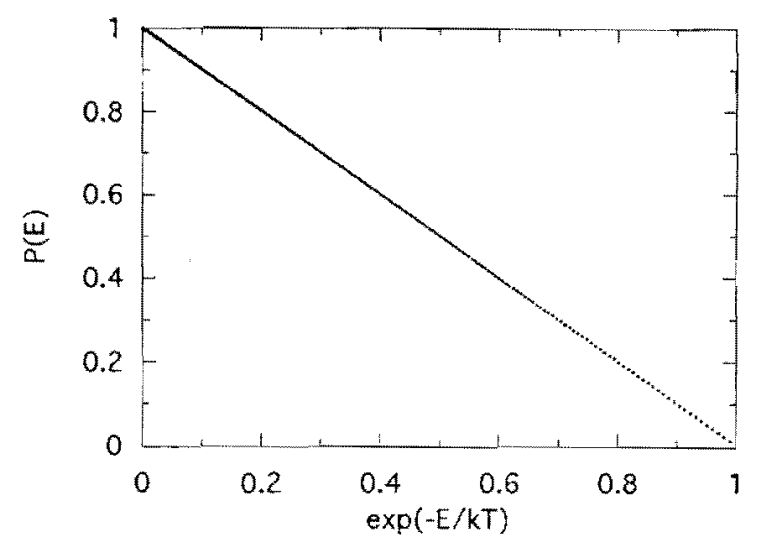

Fig. 2. Probability that the Hamiltonian, for a two-thermostat one-dimensional harmonic oscillator, is less than $E_{\text {, as a function }}$ of $e^{-E}$. There are no statistically significant deviations from the straight line relation, also shown, characteristic of the canonical distribution. The oscillator mass, force constant, temperature, and relaxation times have all been set equal to unity.

oscillator with simultaneous control of both $K$ and $K^{2}$. The equations of motion for this last case (iii) are as follows,

$\dot{q}=p, \quad \dot{p}=-q-\zeta p-\xi p^{3}$,

$\dot{\zeta}=\left(p^{2}-1\right) / \tau_{\zeta}^{2}, \quad \dot{\xi}=\left(p^{4}-3 p^{2}\right) / \tau_{\xi}^{2}$.

Numerical evidence that these equations can provide effectively ergodic coverage of the oscillator phase space is quite strong. The zeroth and sixth moments are given correctly, within statistical fluctuations, for a variety of values of the characteristic times, $\tau_{\xi}$ and $\tau_{\xi}$. The phase-space distribution exhibits no obvious gaps when both moments are controlled. In Fig. 2 we show the cumulative energy distribution for the twothermostat case. There is no significant deviation from the canonical distribution. A study of the angular dependence in $\{q, p\}$ space shows no statistically significant deviation from the expected circular symmetry.

For the case that all system parameters are set equal to unity, our numerical evidence is based on several runs of from $10^{7}$ to $10^{8}$ fourth-order Runge-Kutta time steps of 0.005 each. Larger time steps can lead to instability for runs of this length. Of course, any equations consistent with the canonical distribution must eventually lead to instability for a fixed time step, because the distribution includes momenta that are arbitrarily large. Attempts to in- clude also the sixth moment in the motion equations promptly lead to instability for much smaller timesteps. Evidently specifying two moments is sufficient to provide good phase-space coverage.

\subsection{Many-body flows}

We investigated the same thermostatting ideas for two two-dimensional systems, a cold equilibrium crystal, at about $1 / 40$ th the melting temperature, and a homogeneous far-from-equilibrium fluid shear flow. The extended Gibbs' distribution for \# degrees of freedom contains multiplicative Gaussian distributions for the two friction coefficients,

$f \propto \exp \left[-\frac{1}{2}\left(\# \tau^{2} \zeta^{2}\right)\right] \exp \left[-\frac{1}{2}\left(\# \tau^{2} \xi^{2}\right)\right]$.

Both the equilibrium and nonequilibrium simulations behave well with either centered-difference or Runge-Kutta time steps of the usual size and with relaxation times, $\tau_{\zeta}$ and $\tau_{\xi}$, corresponding to oscillation frequencies of the order of the Einstein frequency $[14], \omega_{\text {Einstein }}=1 / \tau$.

The equations of motion for the shear-flow problem have the form:

$\dot{x}=p_{x}+\dot{\varepsilon} y, \quad \dot{y}=p_{y}, \quad \#=2 N$,

$\dot{p}_{x}=F_{x}-\dot{\varepsilon} p_{y}-\zeta p_{x}-\xi\left(K / K_{0}\right) p_{x}$,

$\dot{p}_{y}=F_{y}-\zeta p_{y}-\xi\left(K / K_{0}\right) p_{y}$,

$\dot{\zeta}=\left(1 / \tau^{2}\right)\left(K / K_{0}-1\right)$,

$\dot{\xi}=\left(1 / \tau^{2}\right)\left(K / K_{0}\right)\left(K / K_{0}-1-2 / \#\right)$.

Provided that the initial center-of-mass velocity vanishes, these equations of motion preserve it unchanged and at the same time reproduce the canonical-ensemble average values of the kinetic energy and its square. The resulting high-density highstrain-rate shear viscosity was consistent with recent constant-energy simulations carried out by Hoover and Posch [15]. Three separate calculations, using $\zeta$ and $\xi$ separately and together, all $10^{6}$ timesteps in length, provided statistically indistinguishable viscosities,

$\{\zeta, \xi, \zeta \xi\} \Rightarrow\{1.14,1.14,1.15\}$

The stabilities and good agreement among these nonequilibrium simulations (the density, tempera- 
ture, and strain rate were all set equal to unity in the reduced units of Ref. [15]) show that it is possible to specify both the kinetic temperature, and its r.m.s. fluctuation, far from equilibrium, and that the constitutive effects of these constraints are relatively small.

\section{Conclusions}

Nosé's mechanics, and some of its various nonHamiltonian generalizations, have proved useful in both equilibrium and nonequilibrium simulations, as described in the references. We believe that the present approach is the simplest of these, as it provides the complete canonical distribution for a harmonic oscillator with two additive thermostatting forces. We have shown that this approach applies also to more usual many-body equilibrium and nonequilibrium problems, though simple temparature control is typically sufficient for these.

The number of variables required to define a nonequilibrium state has been inconclusively discussed for decades. For instance, at physical boundaries either temperatures or heat fluxes can be specified. Energy and stress could be used rather than temperature, density, and strain rate. Again the only lasting criteria are Occam's. To us it seems desirable that solutions in the vicinity of boundaries be as smooth and free of discontinuities as is possible. For this purpose, feedback, which retains time correlations and avoids trajectory discontinuities seems specially valuable.

Evans and Holian [11] have shown that a wide variety of nonequilibrium thermostats leads to sensibly identical behavior far from equilibrium, so that the details of the specified list of independent variables have an academic flavor. Nevertheless it is important to have numerical simulation methods, such as the kinetic moments method described here, which not only reproduce Gibbs' equilibrium statistical ensembles, but also can be extended to nonequilibrium systems.. The properties of time-reversibility and determinism are desirable too, both for the portability and reproducibility of simulations, as well as for the analytic connection between such simulations and the second law of thermodynamics [16].

\section{Acknowledgement}

Carol Hoover and Vic Castillo (LLNL) as well as Harald Posch (U Wien) provided helpful suggestions. A part of this work was performed at the Los Alamos and Lawrence Livermore National Laboratories by the University of California under the auspices of the United States Department of Energy pursuant to Contracts W-7405-Eng-36 and W-7405Eng-48.

\section{References}

[1] S. Nose, J. Chem. Phys. 81 (1984) 511; Molec. Phys. 52 (1984) 255; Prog. Theor. Phys. Suppl. 103 (1991) 1.

[2] W.G. Hoover, Phys. Rev. A 31 (1985) 1695; H.A. Posch, W.G. Hoover and F.J. Vesely, Phys. Rev. A 33 (1986) 4253.

[3] J. Jellinek, J. Chem. Phys. 92 (1988) 3163; J. Jellinek and R.S. Berry, Phys. Rev. A 38 (1988) 3069; 40 (1989) 2816.

[4] W.G. Hoover, Phys. Rev. A 40 (1989) 2814.

[5] A. Bulgac and D Kusnezov, Phys. Rev. A 42 (1990) 5045; D. Kusnezov, A. Bulgac and W. Bauer, Ann. Phys. (NY) 204 (1990) 155.

[6] W.G. Hoover, Computational statistical mechanics (Elsevier, Amsterdam, 1991).

[7] I.P. Hamilton, Phys. Rev. A 42 (1990) 7467.

[8] R.G. Winkler, Phys. Rev. A 45 (1992) 2250.

[9] G.J. Martyna, M.L. Klein and M.E. Tuckerman, J. Chem. Phys. 97 (1992) 2635.

[10] W.G. Hoover, H.A. Posch and L.W. Campbell, Chaos 3 (1993) 325.

[11] DJ. Evans and B.L. Holian, J. Chem. Phys. 83 (1985) 1069.

[12] W.G. Hoover, Phys. Lett. A 204 (1995) 133.

[13] H.J.C. Berendsen and W.F, van Gunsteren, in: Proc. Enrico Fermi School on Molecular-dynamics simulation of statistical mechanical systems, Varenna, eds. G.P.F. Ciccotti and W.G. Hoover (North-Holland, Amsterdam, 1986); G.D. Venneri and W.G. Hoover, J. Comp. Phys. 73 (1987) 468 .

[14] B.L. Holian, A.F. Voter and R. Ravelo, Phys. Rev. E 52 (1995), to be published.

[15] W.G. Hoover and H.A. Posch, Phys. Rev. E 51 (1995) 273.

[16] B.L. Holian, W.G. Hoover and H.A. Posch, Phys. Rev. Lett. $59(1987) 10$. 